\title{
Certain Geometric Properties and Matrix Transformations on a Newly Introduced Banach Space
}

\author{
Merve İkhan ${ }^{1}$ \\ ${ }^{1}$ Department of Mathematics, Faculty of Science and Arts, Düzce University, Düzce, Turkey
}

\section{Article Info}

Keywords: Banach-Saks type p, Fixed point property, Matrix transformations 2010 AMS: 46B45, 46B20, 46A45

Received: 16 April 2020

Accepted: 31 May 2020

Available online: 10 June 2020

\begin{abstract}
The main purpose of this study is to characterize some matrix classes from classical sequence spaces into a newly introduced space and find the norm of some special matrix operators. Also, we give certain geometric properties of this space.
\end{abstract}

\section{Introduction}

The matrix transformations in sequence spaces have been studied by many authors over years. Since the most general linear operators from a sequence space to another one can be given by an infinite matrix, the theory of matrix transformations has been of great importance in the study of sequence spaces. For the relevant literature consult to [1]-[6].

In the recent times, the interest in investigating geometric properties of sequence spaces with topological properties have increased. Over years several papers on the geometric properties of various spaces have appeared. For instance, Mursaleen et al. [7] examined the geometric properties of Euler sequence space. More information about the relevant literature can be found in [8]-[14].

The main purpose of this work is to characterize some matrix classes on a newly introduced sequence space and find the norm of certain bounded linear matrix operators. Also, we prove that the resulting space is of type $p$ Banach-Saks and it has the weak fixed point property. Finally, we investigate the strictly convexity and uniformly convexity of this space.

\section{Preliminaries and notations}

A sequence space is a linear subspace of the space of all real valued sequences $\omega . \ell_{\infty}, c, c_{0}$ and $\ell_{p}(1 \leq p<\infty)$ are the sequence spaces of all bounded, convergent, null sequences and absolutely $p$-summable sequences, respectively.

Given any sequence spaces $X$ and $Y$ and an infinite matrix $T=\left(t_{i j}\right), T$ is called a matrix mapping from $X$ into $Y$ if for every sequence $x=\left(x_{j}\right) \in X, T x=\left(T_{i}(x)\right)$ with

$$
T_{i}(x)=\sum_{j=1}^{\infty} t_{i j} x_{j}
$$

is in $Y$ and the series is convergent for each $i \in \mathbb{N}=\{1,2, \ldots\}$. Then, $T x$ is called the $T$-transform of $x$. 
The set

$$
X_{T}=\left\{x=\left(x_{j}\right) \in \omega: T x \in X\right\}
$$

is called the matrix domain of $T$ in the space $X$ and it is also a sequence space.

Let $\varphi: \mathbb{N} \rightarrow \mathbb{N}$ be the Euler function defined as

$$
\varphi(i)=\sum_{j=1,(j, i)=1}^{i} 1,
$$

where $(j, i)$ is the greatest common divisor of $j$ and $i$. That is, $\varphi(i)$ gives the number of positive integers less than $i$ which are coprime with $j$.

The Euler function $\varphi$ satisfies the following properties:

1. $i=\sum_{j \mid i} \varphi(j)$ holds for every $i \in \mathbb{N}$.

2. $\varphi(i)=i \prod_{p \mid i}\left(1-\frac{1}{p}\right)$, where $p$ is the prime divisor of $i$.

3. $\varphi(i j)=\varphi(i) \varphi(j)$ holds for $(i, j)=1$.

Let $i=p_{1}^{\alpha_{1}} p_{2}^{\alpha_{2}} \ldots p_{l}^{\alpha_{l}}$. The Möbius function $\mu: \mathbb{N} \rightarrow\{-1,0,1\}$ is defined as

$$
\begin{gathered}
\mu(i)=(-1)^{l} \text { if } \alpha_{1}=\alpha_{2}=\ldots=\alpha_{l}=1 \\
\mu(i)=0 \text { if } \alpha_{k} \neq 1 \text { for at least one } k \in\{1,2, \ldots, l\},
\end{gathered}
$$

where $p_{1}, p_{2}, \ldots, p_{l}$ are non-equivalent prime numbers and $p_{1}^{\alpha_{1}} p_{2}^{\alpha_{2}} \ldots p_{l}^{\alpha_{l}}$ is the prime factorization of $i>1$. Also,

$$
\mu(1)=1
$$

and for $i \neq 1$

$$
\sum_{p \mid i} \mu(p)=0
$$

holds.

$\Phi$-summability was introduced by Schoenberg [15] in order to study the Riemann integrability of a generalized Dirichlet function in $[0,1]$. It is said that a sequence $x=\left(x_{j}\right)$ is $\varphi$-convergent to $l$ if

$$
\lim _{i \rightarrow \infty} \frac{1}{i} \sum_{j \mid i} \varphi(j) x_{j}=l .
$$

Let $\Phi=\left(\phi_{i j}\right)$ be the matrix defined as

$$
\phi_{i j}=\left\{\begin{array}{cll}
\frac{\varphi(j)}{i} & , & \text { if } j \mid i, \\
0 & , & \text { if } j \nmid i .
\end{array}\right.
$$

The regularity of this special matrix is also observed by Schoenberg [15]. This means that the matrix $\Phi$ maps $c$ into $c$ and the limit is preserved.

In [16], by using this matrix, the sequence spaces

$$
\ell_{p}(\Phi)=\left\{x=\left(x_{i}\right) \in \omega: \sum_{i}\left|\frac{1}{i} \sum_{j \mid i} \varphi(j) x_{j}\right|^{p}<\infty\right\} \quad(1 \leq p<\infty)
$$

and

$$
\ell_{\infty}(\Phi)=\left\{x=\left(x_{i}\right) \in \omega: \sup _{i}\left|\frac{1}{i} \sum_{j \mid i} \varphi(j) x_{j}\right|<\infty\right\}
$$

are introduced and proved that these spaces are Banach spaces with the norms

$$
\|x\|_{\ell_{p}(\Phi)}=\left(\sum_{i}\left|\frac{1}{i} \sum_{j \mid i} \varphi(j) x_{j}\right|^{p}\right)^{1 / p} \quad(1 \leq p<\infty)
$$


and

$$
\|x\|_{\ell_{\infty}(\Phi)}=\sup _{i}\left|\frac{1}{i} \sum_{j \mid i} \varphi(j) x_{j}\right|,
$$

respectively.

Unless otherwise stated, $\tilde{x}=\left(\tilde{x}_{i}\right)$ will be the $\Phi$-transform of a sequence $x=\left(x_{i}\right)$, that is,

$$
\tilde{x}_{i}=\Phi_{i}(x)=\frac{1}{i} \sum_{j \mid i} \varphi(j) x_{j}
$$

for all $i \in \mathbb{N}$.

\section{Some matrix transformations and norms of matrix operators}

In this part of the study, we firstly give the characterization of matrix classes $\left(X, \ell_{p}(\Phi)\right)$, where $X \in\left\{\ell_{\infty}, c, c_{0}, \ell_{1}\right\}$ and $1 \leq p \leq \infty$. For this aim, we give the following results, where $\mathscr{F}$ denotes the collection of all finite subsets of $\mathbb{N} . q$ is conjugate of $p$; that is $p^{-1}+q^{-1}=1$ with $1<p, q<\infty$.

Lemma 3.1. [17] Let $1 \leq p<\infty$.

(a) $T=\left(t_{i j}\right) \in\left(\ell_{\infty}, \ell_{p}\right)=\left(c, \ell_{p}\right)=\left(c_{0}, \ell_{p}\right)$ if and only if

$$
\sup _{K \in \mathscr{F}} \sum_{i}\left|\sum_{j \in K} t_{i j}\right|^{p}<\infty .
$$

(b) $T=\left(t_{i j}\right) \in\left(\ell_{1}, \ell_{p}\right)$ if and only if

$$
\sup _{j} \sum_{i}\left|t_{i j}\right|^{p}<\infty
$$

(c) $T=\left(t_{i j}\right) \in\left(\ell_{\infty}, \ell_{\infty}\right)=\left(c, \ell_{\infty}\right)=\left(c_{0}, \ell_{\infty}\right)$ if and only if

$$
\sup _{i} \sum_{j}\left|t_{i j}\right|<\infty
$$

(d) $T=\left(t_{i j}\right) \in\left(\ell_{1}, \ell_{\infty}\right)$ if and only if

$$
\sup _{i, j}\left|t_{i j}\right|<\infty .
$$

Theorem 3.2. Let $1 \leq p<\infty$.

(a) $T=\left(t_{i j}\right) \in\left(\ell_{\infty}, \ell_{p}(\Phi)\right)=\left(c, \ell_{p}(\Phi)\right)=\left(c_{0}, \ell_{p}(\Phi)\right)$ if and only if

$$
\sup _{K \in \mathscr{F}} \sum_{i}\left|\sum_{j \in K} \sum_{l \mid i} \frac{\varphi(l)}{i} t_{l j}\right|^{p}<\infty .
$$

(b) $T=\left(t_{i j}\right) \in\left(\ell_{1}, \ell_{p}(\Phi)\right)$ if and only if

$$
\sup _{j} \sum_{i}\left|\sum_{l \mid i} \frac{\varphi(l)}{i} t_{l j}\right|^{p}<\infty .
$$

(c) $T=\left(t_{i j}\right) \in\left(\ell_{\infty}, \ell_{\infty}(\Phi)\right)=\left(c, \ell_{\infty}(\Phi)\right)=\left(c_{0}, \ell_{\infty}(\Phi)\right)$ if and only if

$$
\sup _{i} \sum_{j}\left|\sum_{l \mid i} \frac{\varphi(l)}{i} t_{l j}\right|<\infty .
$$

(d) $T=\left(t_{i j}\right) \in\left(\ell_{1}, \ell_{\infty}(\Phi)\right)$ if and only if

$$
\sup _{i, j}\left|\sum_{l \mid i} \frac{\varphi(l)}{i} t_{l j}\right|<\infty .
$$


Proof. Given any infinite matrix $T=\left(t_{i j}\right) \in\left(\ell_{\infty}, \ell_{p}(\Phi)\right)$, define a new matrix $\hat{T}=\left(\hat{t}_{i j}\right)$ by

$$
\hat{t}_{i j}=\sum_{l \mid i} \frac{\varphi(l)}{i} t_{l j}
$$

for all $i, j \in \mathbb{N}$. Then, for any $x=\left(x_{j}\right) \in \ell_{\infty}$, the equality

$$
\sum_{j} \hat{t}_{i j} x_{j}=\sum_{l \mid i} \frac{\varphi(l)}{i} \sum_{j} t_{l j} x_{j}
$$

means that $\hat{T}_{i}(x)=\Phi_{i}(T x)$ for all $i \in \mathbb{N}$. This implies that $T x \in \ell_{p}(\Phi)$ for $x=\left(x_{j}\right) \in \ell_{\infty}$ if and only if $\hat{T} x \in \ell_{p}$ for $x=\left(x_{j}\right) \in \ell_{\infty}$. Hence, we conclude from Lemma 3.1 (a) that

$$
\sup _{K \in \mathscr{F}} \sum_{i}\left|\sum_{j \in K} \sum_{l \mid i} \frac{\varphi(l)}{i} t_{l j}\right|^{p}<\infty .
$$

The other results follow with the same technique by using Lemma 3.1 (b), (c) and (d).

Now, we investigate the norm of the bounded linear matrix operators from $\ell_{p}(\Phi)$ into $\ell_{1}(\Phi)$ and $\ell_{\infty}(\Phi)$ for $1 \leq p \leq \infty$. Firstly, we have a lemma which is essential for our investigation.

Lemma 3.3. Given any infinite matrix $T=\left(t_{i j}\right)$, the following statements hold:

(a) The norm of $T \in B\left(\ell_{p}, \ell_{\infty}\right)$ is defined by

$$
\|T\|_{\left(\ell_{1}, \ell_{\infty}\right)}=\sup _{i, j}\left|t_{i j}\right|
$$

and

$$
\|T\|_{\left(\ell_{p}, \ell_{\infty}\right)}=\sup _{i} \sum_{j}\left|t_{i j}\right|^{q} \quad(1<p \leq \infty) .
$$

(b) The norm of $T \in B\left(\ell_{p}, \ell_{1}\right)$ is defined by

$$
\|T\|_{\left(\ell_{1}, \ell_{1}\right)}=\sup _{j} \sum_{i}\left|t_{i j}\right|
$$

and

$$
\|T\|_{\left(\ell_{p}, \ell_{1}\right)}=\sup _{K \in \mathscr{F}} \sum_{j}\left|\sum_{i \in K} t_{i j}\right|^{q} \quad(1<p \leq \infty) .
$$

Theorem 3.4. Let $T=\left(t_{i j}\right)$ be an infinite matrix.

(a) If $T \in B\left(\ell_{1}(\Phi), \ell_{\infty}(\Phi)\right)$, then

$$
A_{1}^{\infty}=\sup _{i, j}\left|\sum_{j \mid l} \frac{\mu\left(\frac{l}{j}\right)}{\varphi(l)} j \sum_{k \mid i} \frac{\varphi(k)}{i} t_{k l}\right|
$$

is finite. In this case, $\|T\|_{\left(\ell_{1}(\Phi), \ell_{\infty}(\Phi)\right)}=A_{1}^{\infty}$.

(b) Let $1<p \leq \infty$. If $T \in B\left(\ell_{p}(\Phi), \ell_{\infty}(\Phi)\right)$, then

$$
A_{p}^{\infty}=\sup _{i} \sum_{j}\left|\sum_{j \mid l} \frac{\mu\left(\frac{l}{j}\right)}{\varphi(l)} j \sum_{k \mid i} \frac{\varphi(k)}{i} t_{k l}\right|^{q}
$$

is finite. In this case, $\|T\|_{\left(\ell_{p}(\Phi), \ell_{\infty}(\Phi)\right)}=A_{p}^{\infty}$.

(c) If $T \in B\left(\ell_{1}(\Phi), \ell_{1}(\Phi)\right)$, then

$$
A_{1}^{1}=\sup _{j} \sum_{i}\left|\sum_{j \mid l} \frac{\mu\left(\frac{l}{j}\right)}{\varphi(l)} j \sum_{k \mid i} \frac{\varphi(k)}{i} t_{k l}\right|
$$

is finite. In this case, $\|T\|_{\left(\ell_{1}(\Phi), \ell_{1}(\Phi)\right)}=A_{1}^{1}$.

(d) Let $1<p \leq \infty$. If $T \in B\left(\ell_{p}(\Phi), \ell_{1}(\Phi)\right)$, then

$$
A_{p}^{1}=\sup _{K \in \mathscr{F}} \sum_{j}\left|\sum_{i \in K} \sum_{j \mid l} \frac{\mu\left(\frac{l}{j}\right)}{\varphi(l)} j \sum_{k \mid i} \frac{\varphi(k)}{i} t_{k l}\right|^{q}
$$

is finite. In this case, $\|T\|_{\left(\ell_{p}(\Phi), \ell_{1}(\Phi)\right)}=A_{p}^{1}$. 
Proof. Let $\tilde{T}=\Phi T \Phi^{-1}$. From Theorem 3 in [16], it is known that the spaces $\ell_{p}(\Phi)$ and $\ell_{p}$ are linearly isomorphic, where $1 \leq p \leq \infty$. Hence, we deduce from the following diagram

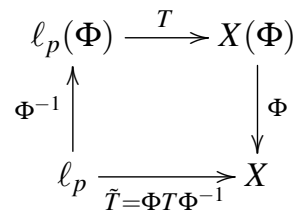

that $\|T\|_{\left(\ell_{p}(\phi), X(\Phi)\right)}=\|\tilde{T}\|_{\left(\ell_{p}, X\right)}$, where $X \in\left\{\ell_{\infty}, \ell_{1}\right\}$ and $1 \leq p \leq \infty$. Thus, the desired results follows from Lemma 3.3.

\section{Certain geometric properties of $\ell_{p}(\Phi)$}

In this part of the study, some geometric properties of the space $\ell_{p}(\Phi)$ for $1<p<\infty$ is given. $\mathscr{B}_{X}$ denotes the unit ball in a normed space $(X,\|\cdot\|)$.

It is said that a Banach space $X$ satisfies the Banach-Saks property if every sequence $\left(u_{n}\right)$ in $X \cap \ell_{\infty}$ has a subsequence $\left(t_{n}\right)$ such that the sequence $\left(a_{k}(t)\right)$ is convergent, where

$$
a_{k}(t)=\frac{1}{k+1}\left(t_{0}+t_{1}+\ldots+t_{k}\right) ;(k \in \mathbb{N}) .
$$

It is said that a Banach space $X$ satisfies the weak Banach-Saks property if there exists a subsequence $\left(t_{n}\right)$ of a given weakly null sequence $\left(u_{n}\right)$ in $X$ such that the sequence $\left(a_{k}(t)\right)$ is strongly convergent to zero.

It is said that a Banach space satisfies the property Banach-Saks type p if every weakly null sequence $\left(u_{k}\right)$ has a subsequence $\left(u_{k_{j}}\right)$ such that for some $C>0$,

$$
\left\|\sum_{j=1}^{n} u_{k_{j}}\right\|<C n^{1 / p}
$$

for all $n \in \mathbb{N}$. Note that $n^{1 / \infty}=1$ for all $n \in \mathbb{N}([18])$.

Theorem 4.1. The space $\ell_{p}(\Phi)$ is of type $p$ Banach-Saks for $1<p<\infty$.

Proof. Let $\left(\delta_{n}\right)$ be a sequence such that $\delta_{n}>0$ for all $n \in \mathbb{N}$ and $\sum_{n} \delta_{n} \leq 1 / 2$. Choose a weakly null sequence $\left(u_{n}\right)$ in $\mathscr{B}_{\ell_{p}}(\Phi)$. Put $t_{1}=u_{n_{1}}=u_{1}$. There exists $m_{1} \in \mathbb{N}$ such that

$$
\left\|\sum_{i=m_{1}+1}^{\infty} t_{1}^{i} \varepsilon^{i}\right\|_{\ell_{p}(\Phi)}<\delta_{1} .
$$

Since $\left(u_{n}\right)$ is weakly null sequence implies $u_{n} \rightarrow 0$ coordinatewise, there is an $n_{2} \in \mathbb{N}$ such that

$$
\left\|\sum_{i=1}^{m_{1}} u_{n}^{i} \varepsilon^{i}\right\|_{\ell_{p}(\Phi)}<\delta_{1},
$$

for all $n \geq n_{2}$. Put $t_{2}=u_{n_{2}}$. Then, there exists an $m_{2}>m_{1}$ such that

$$
\left\|\sum_{i=m_{2}+1}^{\infty} t_{2}^{i} \varepsilon^{i}\right\|_{\ell_{p}(\Phi)}<\delta_{2} .
$$

Again using the fact that $u_{n} \rightarrow 0$ coordinatewise, there exists an $n_{3}>n_{2}$ such that

$$
\left\|\sum_{i=1}^{m_{2}} u_{n}^{i} \varepsilon^{i}\right\|_{\ell_{p}(\Phi)}<\delta_{2}
$$

for all $n \geq n_{3}$.

By continuing this process, we obtain two sequences $\left(m_{i}\right)$ with $m_{1}<m_{2}<\ldots<m_{i}<\ldots$ and $\left(n_{i}\right)$ with $n_{1}<n_{2}<\ldots<n_{i}<\ldots$ such that

$$
\left\|\sum_{i=1}^{m_{j}} u_{n}^{i} \varepsilon^{i}\right\|_{\ell_{p}(\Phi)}<\delta_{j},
$$


for all $n \geq n_{j+1}$ and

$$
\left\|\sum_{i=m_{j}+1}^{\infty} t_{j}^{i} \varepsilon^{i}\right\|_{\ell_{p}(\Phi)}<\delta_{j}
$$

where $t_{j}=u_{n_{j}}$. It follows that

$$
\begin{aligned}
\left\|\sum_{j=1}^{n} t_{j}\right\|_{\ell_{p}(\Phi)} & =\left\|\sum_{j=1}^{n}\left(\sum_{i=1}^{m_{j-1}} t_{j}^{i} \varepsilon^{i}+\sum_{i=m_{j-1}+1}^{m_{j}} t_{j}^{i} \varepsilon^{i}+\sum_{i=m_{j}+1}^{\infty} t_{j}^{i} \varepsilon^{i}\right)\right\|_{\ell_{p}(\Phi)} \\
& \leq\left\|\sum_{j=1}^{n}\left(\sum_{i=m_{j-1}+1}^{m_{j}} t_{j}^{i} \varepsilon^{i}\right)\right\|_{\ell_{p}(\Phi)}+2 \sum_{j=0}^{n} \delta_{j} .
\end{aligned}
$$

Also, given any $u \in \mathscr{B}_{\ell_{p}(\Phi)}$, we have $\|u\|_{\ell_{p}(\Phi)}^{p}=\sum_{i=1}^{\infty}\left|\frac{1}{i} \sum_{k \mid i} \varphi(k) u_{k}\right|^{p}<1$. Therefore, we have that

$$
\begin{aligned}
\left\|\sum_{j=1}^{n}\left(\sum_{i=m_{j-1}+1}^{m_{j}} t_{j}^{i} \varepsilon^{i}\right)\right\|_{\ell_{p}(\Phi)}^{p} & =\sum_{j=1}^{n} \sum_{i=m_{j-1}+1}^{m_{j}}\left|\frac{1}{i} \sum_{k \mid i} \varphi(k) t_{j}^{k}\right|^{p} \\
& \leq \sum_{j=1}^{n} \sum_{i=1}^{\infty}\left|\frac{1}{i} \sum_{k \mid i} \varphi(k) t_{j}^{k}\right|^{p} \leq n
\end{aligned}
$$

Hence, we obtain

$$
\left\|\sum_{j=1}^{n}\left(\sum_{i=m_{j-1}+1}^{m_{j}} t_{j}^{i} \varepsilon^{i}\right)\right\|_{\ell_{p}(\Phi)} \leq n^{1 / p}
$$

Since $n^{1 / p} \geq 1$ holds for all $n \in \mathbb{N}$ and $1<p<\infty$, we have

$$
\left\|\sum_{j=1}^{n} t_{j}\right\|_{\ell_{p}(\Phi)} \leq n^{1 / p}+1 \leq 2 n^{1 / p}
$$

Hence, we conclude that $\ell_{p}(\Phi)$ is of type $p$ Banach-Saks for $1<p<\infty$.

García-Falset [19] introduce the following coefficient:

$$
R(X)=\sup \left\{\liminf _{n \rightarrow \infty}\left\|u_{n}-L\right\|:\left(u_{n}\right) \text { is a sequence in } \mathscr{B}_{X}, u_{n} \stackrel{w}{\rightarrow} 0, L \in \mathscr{B}_{X}\right\} .
$$

Here $u_{n} \stackrel{w}{\rightarrow} 0$ means that $\left(u_{n}\right)$ is weakly convergent to zero. A Banach space $X$ with $R(X)<2$ has the weak fixed point property ([20]).

Remark 4.2. $R\left(\ell_{p}(\Phi)\right)=R\left(\ell_{p}\right)=2^{1 / p}$ since $\ell_{p}(\Phi)$ is linearly isomorphic to $\ell_{p}$.

Hence, we have the following result.

Theorem 4.3. The space $\ell_{p}(\Phi)$ has the weak fixed point property for $1<p<\infty$.

Let $\mathscr{S}_{X}=\{u \in X:\|u\|=1\}$. The Gurarii's modulus of convexity is

$$
\beta_{X}(\delta)=\inf \left\{1-\inf _{0 \leq \lambda \leq 1}\|\lambda u+(1-\lambda) v\|: u, v \in \mathscr{S}_{X},\|u-v\|=\delta\right\}
$$

where $0 \leq \delta \leq 2$ ([21]).

Theorem 4.4. The inequality $\beta_{\ell_{p}(\Phi)}(\delta) \leq 1-\left[1-\left(\frac{\delta}{2}\right)^{p}\right]^{1 / p}$ holds, where $0 \leq \delta \leq 2$.

Proof. Let $0 \leq \delta \leq 2$. Consider the sequences

$$
\tilde{u}=\left(\left(1-\left(\frac{\delta}{2}\right)^{p}\right)^{1 / p}, \frac{\delta}{2}, 0,0,0, \ldots\right)
$$


and

$$
\tilde{v}=\left(\left(1-\left(\frac{\delta}{2}\right)^{p}\right)^{1 / p},-\frac{\delta}{2}, 0,0,0, \ldots\right) .
$$

Set $u=\Phi^{-1} \tilde{u}$ and $v=\Phi^{-1} \tilde{v}$. By using the relation (2.1), we obtain that

$$
\|u\|_{\ell_{p}(\Phi)}^{p}=\|\Phi u\|_{\ell_{p}}^{p}=\|\tilde{u}\|_{\ell_{p}}^{p}=\left|\left(1-\left(\frac{\delta}{2}\right)^{p}\right)^{1 / p}\right|^{p}+\left|\frac{\delta}{2}\right|^{p}=1
$$

and

$$
\|v\|_{\ell_{p}(\Phi)}^{p}=\|\Phi v\|_{\ell_{p}}^{p}=\|\tilde{v}\|_{\ell_{p}}^{p}=\left|\left(1-\left(\frac{\delta}{2}\right)^{p}\right)^{1 / p}\right|^{p}+\left|-\frac{\delta}{2}\right|^{p}=1 .
$$

Also, we have

$$
\|u-v\|_{\ell_{p}(\Phi)}^{p}=\|\tilde{u}-\tilde{v}\|_{\ell_{p}}^{p}=\left(|\delta|^{p}\right)^{1 / p}=\delta
$$

Hence, we conclude that

$$
\begin{aligned}
\beta_{\ell_{p}(\Phi)}(\delta) & \leq 1-\inf _{0 \leq \lambda \leq 1}\|\lambda u+(1-\lambda) v\|_{\ell_{p}(\Phi)} \\
& \leq 1-\inf _{0 \leq \lambda \leq 1}\|\lambda \tilde{u}+(1-\lambda) \tilde{v}\|_{\ell_{p}} \\
& \leq 1-\inf _{0 \leq \lambda \leq 1}\left[\left|\lambda\left(1-\left(\frac{\delta}{2}\right)^{p}\right)^{1 / p}+(1-\lambda)\left(1-\left(\frac{\delta}{2}\right)^{p}\right)^{1 / p}\right|^{p}+\left|\lambda \frac{\delta}{2}-(1-\lambda) \frac{\delta}{2}\right|^{p}\right]^{1 / p} \\
& \leq 1-\inf _{0 \leq \lambda \leq 1}\left[1-\left(\frac{\delta}{2}\right)^{p}+|2 \lambda-1|^{p} \frac{\delta^{p}}{2}\right]^{1 / p} \\
& \leq 1-\left[1-\left(\frac{\delta}{2}\right)^{p}\right]^{1 / p} .
\end{aligned}
$$

Corollary 4.5. If $\beta_{\ell_{p}(\Phi)}(\delta)=1$, then $\ell_{p}(\Phi)$ is strictly convex.

Corollary 4.6. If $0<\beta_{\ell_{p}(\Phi)}(\delta) \leq 1$, then $\ell_{p}(\Phi)$ is uniformly convex.

\section{References}

[1] F. Basar, Summability Theory and Its Applications, Bentham Science Publishers, İstanbul, 2012.

[2] M. Et, On some difference sequence spaces, Turkish J. Math., 17 (1993), 18-24.

[3] E. E. Kara, M. Başarır, On compact operators and some Euler B ${ }^{(m)}$ difference sequence spaces, J. Math. Anal. Appl., 379(2) (2011), 499-511.

[4] M. Kirişçi, F. Başar, Some new sequence spaces derived by the domain of generalized difference matrix, Comput. Math. Appl., 60 (2010), $1299-1309$.

[5] M. Mursaleen, A. K. Noman, On the spaces of $\lambda$-convergent and bounded sequences, Thai J. Math., 8(2) (2012), 311-329.

[6] P. Zengin Alp, M. İlkhan, On the difference sequence space $\ell_{p}\left(\hat{T}^{q}\right)$, Math. Sci. Appl. E-Notes, 7(2) (2019), 161-173.

[7] M. Mursaleen, F. Başar, B. Altay, On the Euler sequence spaces which include the spaces $\ell_{p}$ and $\ell_{\infty} I I$, Nonlinear Anal., 65(3) (2006), 707-717.

[8] S. Demiriz, C. Çakan, Some topological and geometrical properties of a new difference sequence space, Abstr. Appl. Anal., 2011 (2011), Article ID 213878,14 pages.

[9] M. Et, M. Karakaş, V. Karakaya, Some geometric properties of a new difference sequence space defined by de la Vallee-Poussin mean, Appl. Math. Comput., 234 (2014), 237-244.

[10] E. E. Kara, Some topological and geometrical properties of new Banach sequence spaces, J. Inequal. Appl., 2013 (2013), 38,15 pages.

[11] V. Karakaya, Some geometric properties of sequence spaces involving lacunary sequence, J. Inequal. Appl., 2007 (2007), Article ID 81028, 8 pages.

[12] E. Savaş, V. Karakaya, N. Şimşek, Some $\ell(p)$-type new sequence spaces and their geometric properties, Abstr. Appl. Anal., 2009 (2009), Article ID 696971,12 pages.

[13] S. Suantai, On the H-property of some Banach sequence spaces, Arch. Math., 39(4) (2003), 309-316.

[14] N. Şimşek, V. Karakaya, On some geometrical properties of generalized modular spaces of Cesaro type defined by weighted means, J. Inequal. Appl., 2009 (2009), Article ID 932734, 13 pages.

[15] I. Schoenberg, The integrability of certain functions and related summability methods, Amer. Math. Monthly, 66 (1959), $361-375$.

[16] M. İlkhan, E. E. Kara, A new Banach space defined by Euler totient matrix operator, Oper. Matrices, 13(2) (2019), 527-544.

[17] M. Stieglitz, H. Tietz, Matrix transformationen von folgenraumen eine ergebnisübersicht, Math. Z., 154 (1977), 1-16.

[18] H. Knaust, Orlicz sequence spaces of Banach-Saks type, Arch. Math., 59(6) (1992), 562-565.

[19] J. García-Falset, Stability and fixed points for nonexpansive mappings, Houston J. Math., 20(3) (1994), $495-506$.

[20] J. García-Falset, The fixed point property in Banach spaces with the NUS-property, J. Math. Anal. Appl., 215(2) (1997), 532-542.

[21] L. Sànchez, A. Ullàn, Some properties of Gurarii’s modulus of convexity, Arch. Math., 71 (1998), 399-406. 